\title{
Renyi entropy for the doped graphene at low temperatures
}

\author{
N. N. Konobeeva ${ }^{1}$, A. A. Polunina ${ }^{1}$, M. B. Belonenko ${ }^{1,2}$ \\ ${ }^{1}$ Volgograd State University, Volgograd, Russia \\ ${ }^{2}$ Volgograd Institute of Business, Volgograd, Russia \\ yana_nn@inbox.ru,polunina_a@mail.ru,mbelonenko@yandex.ru
}

PACS 72.80.Vp, 73.22.Pr

DOI 10.17586/2220-8054-2015-6-2-274-279

The distribution function for the perimeter of a simply connected cluster containing undoped lattice sites is based on percolation theory and the hypothesis of scale invariance. The Renyi entropy for doped graphene at low temperatures was calculated on the basis of this distribution function.

Keywords: nanostructure, graphene, Renyi entropy.

Received: 24 November 2014

Revised: 2 December 2014

Final revision: 16 December 2014

\section{Introduction}

Renyi entropy plays an important role in various fields of human activity: in ecology and statistics as the diversity index, in quantum information - as a measure of complexity, in statistical mechanics - to describe quantum dissipative systems [1,2]. The most important areas for the application of entropy are quantum-mechanical and relativistic objects. The applications of entropy are of great interest in quantum physics and astrophysics. It is just enough to merely mention one of the most important results of black hole thermodynamics: the entropy of a black hole is equal to one quarter of its surface area (the area of the event horizon) (Hawking, 1978) [3].The broad appeal of the formulas of the type $s \sim k \sum p \log (p)$ in different areas may be linked to the special role of power laws in nature [4]. For such cases, the expression $p \log (p)$ is a convenient additive measure. Many scientific papers concerning Renyi entropy and entanglement have been published recently [5] - the phenomenon in which quantum state objects are interdependent even passing between them through space. The spectral complexity and entropy concept is becoming a powerful tool to gain access to such universal values as Central charge, which is associated with the conformal field theory and used to describe the main conditions and their degeneration. This effect can also be useful for quantum computing or quantum cryptography.

It should be noted that graphene electrons near the Fermi level are described with the conformal field theory (in this case the Dirac equations reduce to the Laplace equation). Additionally, technology development allows one to form a graphene field with different boundaries (including limited boundaries) [6-8], which makes possible experimental verification of the consequences of the conformal theories and of the applications to quantum information theory. 


\section{The statement of the problem}

The main result of the use of conformal field theory states that the Renyi entropy can be found from the formula [9]:

$$
s_{n}=\frac{n+1}{6 n} \ln \left(\frac{L}{a}\right),
$$

here, $a$ is the lattice constant, $L$ is the perimeter of the closed path (in units of a), $\mathrm{n}$ is the order of entropy. Higher values of $n$, directing to infinity, give the Renyi entropy, which is greatly determined by considering only the highest probability events. Lower values of $n$, tending to zero, give the Renyi entropy, which increasingly considers all possible events more uniformly, regardless their probabilities.

After averaging according to the size of the cluster, formula (1), under the assumption of the power distribution function, gives:

$$
s_{n}=\frac{n+1}{6 n} \int_{a}^{\infty} \ln \left(\frac{L}{a}\right)\left(\frac{L}{a}\right)^{\beta} d L .
$$

This integral converges when $\beta \leqslant-2$.

Here, it is necessary to make a major retreat. It is possible to describe the behavior of the main variables by a power law (it is well-known fact from the theory of phase transitions in the critical region near the phase transition point where the fluctuations are particularly large). The corresponding indicators are called critical exponents. Such behavior has been obtained in the framework of scaling theory, and other approaches. Actually, the existence of scaling [10] means that in the system (in the area near-critical), there is no allocated length scale and fluctuations can have an arbitrarily large amount of spatial correlation. So, it is reasonable to assume that the dependence of the distribution function upon the perimeter will be of power-law form, with the index, which will be defined below.

It should be noted that the power distribution function follows from scale invariance theory. The scale invariance theory is based on a power dependence of some properties, and the exponent depends on the dimension of space and the symmetry of the system [11].Thus, the calculation of the Renyi entropy is reduced to the problem of determining the index $\beta$. This has been solved by modeling methods.

The principle of modeling distribution of admixtures on the surface of a hexagonal lattice of a graphene was used in this case (Fig. 1). It was assumed that the electron cannot access the area (nodes, node) occupied by the admixture. In areas which are not occupied by admixtures, electron motion is described by the well-known Dirac equation for graphene [12]:

$$
H \psi=i \hbar \frac{d \psi}{d t}, H=-i \hbar v_{f} \bar{\sigma} \cdot \bar{\nabla} .
$$

At the Fermi level, this equation is reduced to the conformal theory with the central charge $\mathrm{c}=1$.

\section{The simulation of the confined areas}

Electrons in graphene have a band structure and move on the lattice sites freely [13]. However, with the appearance in the graphene lattice sites of impurity atoms forming carbon bond, the site becomes unavailable for an electron, which is located in the conduction band. I.e. there is a border, and in the case of the first approximation value of the potential barrier for electrons can be considered as infinity, because of the site unavailability. In the wave approximation, the modeling problem of electron motion was solved in paper [14]. It is 
worth noting that electron motion at the lattice sites does not distort the bond lengths or local charge density, since the electron is already in the conduction band (i.e., electron is delocalized).

It should also be noted that the units engaged with impurity atoms are unavailable for electrons. This leads to distortion of the band structure. For example, when an electron moves in a limited area, which is described by equation (3), there are discrete energy levels and not all values of the electron momentum are allowed. The remaining electron in the conduction band, because of the reflection of the "walls" formed by impurity atoms and further interference, can have only certain values of momentum and energy. The closest analogue is a classic resonator which carves out a certain frequency range. Since the impurities are randomly distributed and the border region has a complex shape, in the general case, the spectrum is continuous but there are features in the density of states described by Anderson [15].

The points of intersection of the lines are called nodes, the lines will be called links or edges. The confined areas, made up of relations - clusters, otherwise are called the corrals.

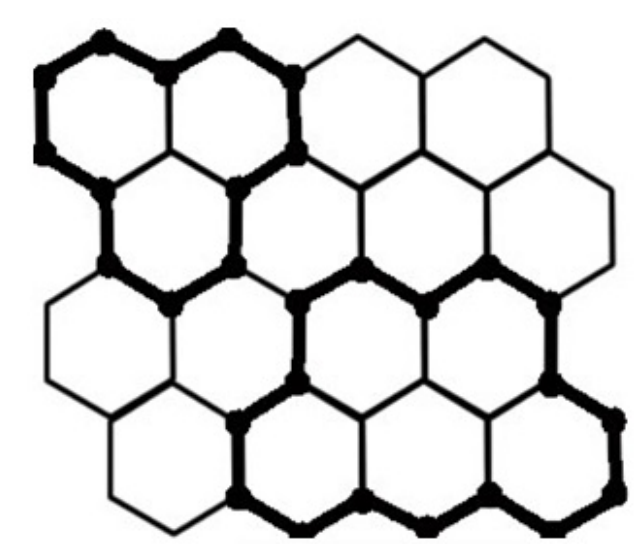

FIG. 1. Confined clusters

To find the clusters, we will solve the problem of relations, that is, we will need to store information about the edges, but not about the actual nodes.

The first stage of simulation is to place admixtures in the nodes of a hexagonal lattice. Since the percolation threshold for a hexagonal lattice is - 0.67 , then the total number of nodes occupied by the electrons should be equal to $67 \%$ or even higher. search).

The algorithm for finding the corrals is based on Lee's algorithm (breadth-first

Figure 2 shows the initial distribution of admixtures and the found endless paths after performing the algorithm.

At the next stage, the connection between the number of closed paths and the length of the closed path was shown. After approximation, it has the form (Figure 3).

This dependence is approximated by the formula:

$$
y \sim x^{-2.97}
$$

and gives $\beta \simeq-3$. 


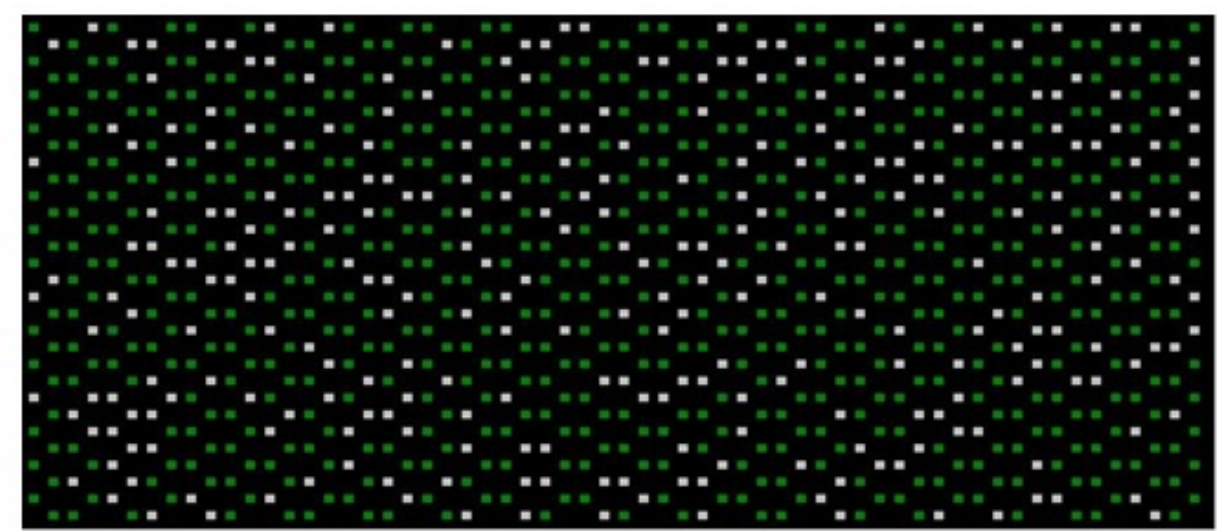

(A)

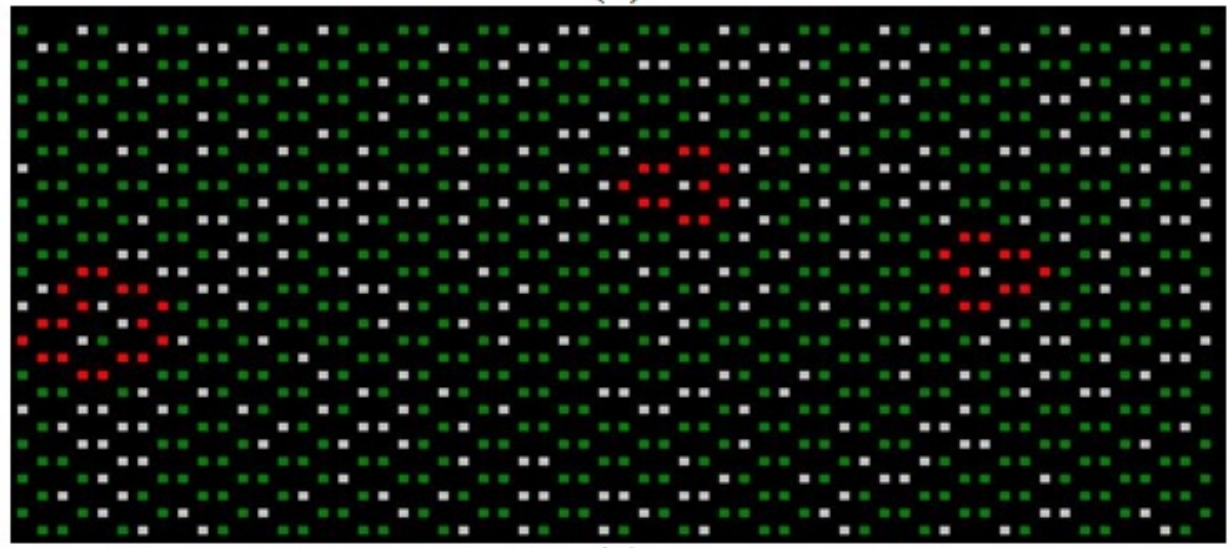

(B)

FIG. 2. The distribution of electrons on a hexagonal lattice: (A) before performing the algorithm; (B) after performing the algorithm

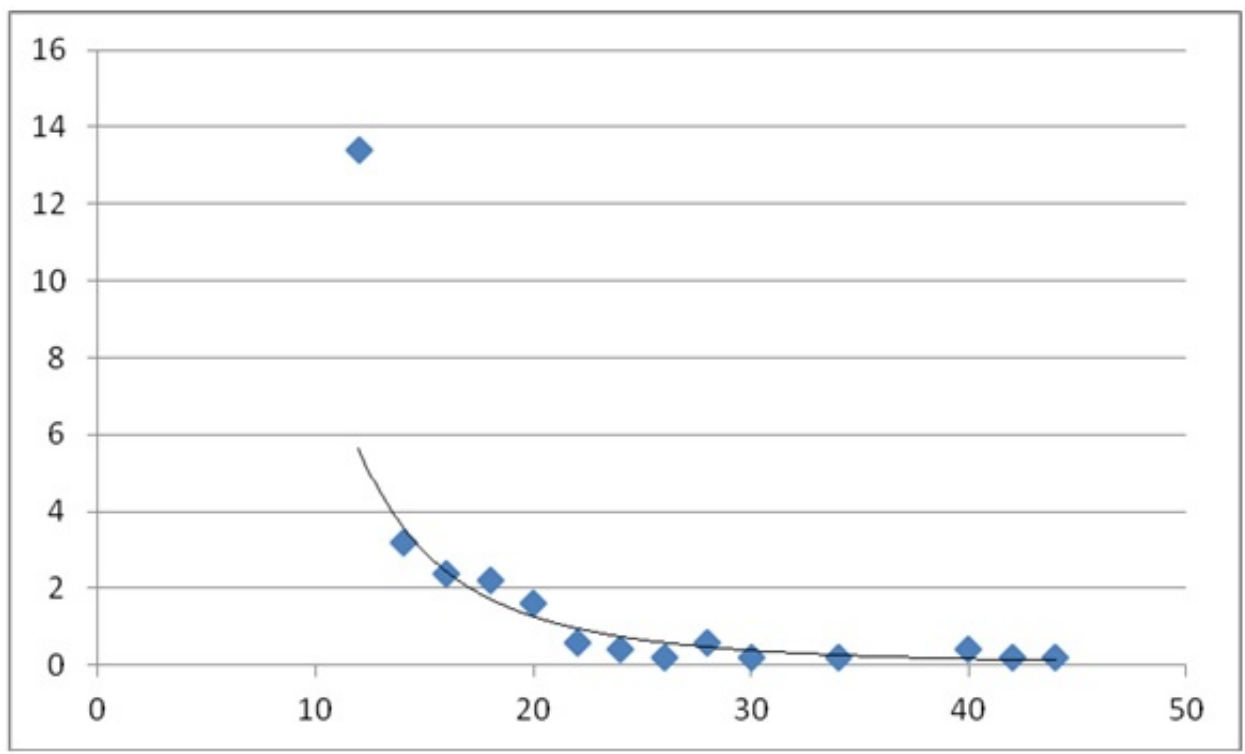

FIG. 3. A plot of the number of closed paths of the length of the closed path 


\section{The summary and conclusions}

In our case, if $\beta \simeq-3$, we obtain the following expression for Renyi entropy:

$$
s_{n}=\frac{n+1}{24 n} .
$$

The dependence of the Renyi entropy of its order is shown in Figure 4.

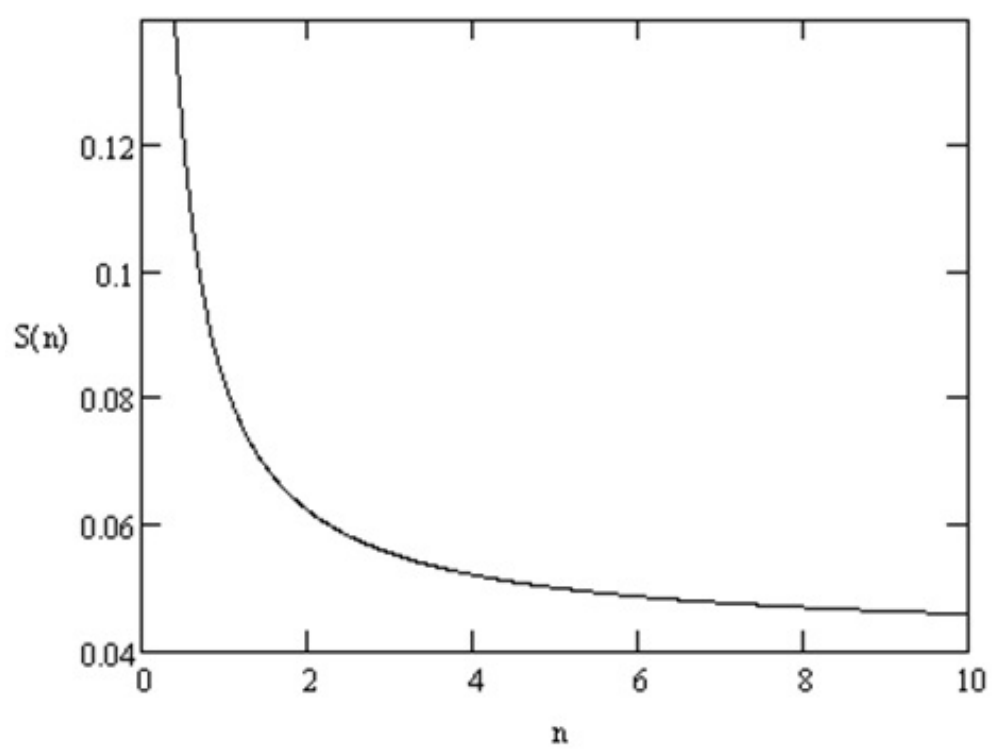

FIG. 4. The dependence of the Renyi entropy upon its order

In conclusion, we will now formulate the main outcomes of this work.

(1) Based on the algorithm of Lee, an algorithm was developed for finding the closed paths containing the electrons of the admixture;

(2) A dependence was constructed for the number of closed paths from their length near the percolation threshold.

(3) The form of the power function was determined, which allowed calculation of the Renyi entropy for doped graphene.

(4) The obtained results can be used to determine the thermodynamic characteristics of doped graphene.

\section{Acknowledgments}

This work was supported by the Russian Foundation for Basic Research under project No. 12-02-31654.

\section{References}

[1] Kirchanov V.S. Using the Renyi entropy to describe quantum dissipative systems in statistical mechanics. Theoretical and Mathematical Physics, 156(3), P. 444-453 (2008).

[2] Chumak O.V. Entropy and fractals in data analysis. Moscow, Izhevsk: SRC "Regular and Chaotic Dynamics", Institute of Computer Science, 164 p. (2011).

[3] Hawking S.W. Black hole and thermodynamics collection of articles "Black hole". News of fundamental physics. Issue 9. P. 204-221 (1978).

[4] Schroeder M. Fractals, Chaos, Power Laws. Moscow, Izhevsk: R\&C Dynamics, 527 p. (2001).

[5] Assaad F.F., Lang T.C., Parisen Toldin F. Entanglement Spectra of Interacting Fermions in Quantum Monte Carlo Simulations (2013). (http://arxiv.org/pdf/1311.5851.pdf). 
[6] Xiong H., Wu B. Atomic Quantum Corrals for Bose-Einstein Condensates. Phys. Rev. A., 82, P. 053634 (2010).

[7] Rossi E., Morr D.K. Spatially dependent Kondo effect in Quantum Corrals. Phys. Rev. Lett., 97, P. $236602(2006)$.

[8] Novoselov K.S., Geim A.K., Morozov S.V., Jiang D., Katsnelson M.I., Grigorieva I.V., Firsov A.A. Two-dimensional gas of massless Dirac fermions in graphene. Nature, 438, P. 197 (2005).

[9] Datta S., David J.R. Renyi entropies of free bosons on the torus and holography (2013). (http://arxiv.org/pdf/1311.1218.pdf).

[10] Patashinskii A.Z., Pokrovskii V.L. Fluctuation theory of phase transitions. Moscow: Nauka, 381 p. (1982).

[11] Müller H. Scaling as a fundamental property of natural vibrations of matter and the fractal structure of space-time. Foundations of physics and geometry. P. 189-209 (2008).

[12] Gusynin V. P., Shaparov S.G., Carbotte J.P. AC conductivity of graphene: from tight-binding model to 2+1-dimensional quantum electrodynamics. Int. J. Mod. Phys. B., 21, P. 4611-4658 (2007).

[13] Castro Neto A.H., Guinea F., Peres N.M.R., Novoselov K.S., Geim A.K. The electronic properties of graphene. Rev. Mod. Phys., 81, P. 109 (2009).

[14] Logemann R., Reijnders K.J.A., Tudorovskiy T., Katsnelson M.I., Yuan S. Modeling Klein Tunneling and Caustics of Electron Waves in Graphene (2014). (http://arxiv.org/pdf/1409.1277v1.pdf).

[15] Gantmaher V.F. Electrons in disordered media. Moscow: Fizmatlit, 288 p. (2013). 